\title{
Kaposi Sarcoma in HIV Positive Nigerian Children: A Case Series
}

\author{
Regina Oladokun $^{1 *}$, Bamidele Kolude ${ }^{2}$, Gabriel Ogun ${ }^{3}$, Biobele Brown ${ }^{1}$, Kikelomo Osinusi $^{1}$ \\ ${ }^{1}$ Department of Paediatrics, University of Ibadan and University College Hospital Ibadan, Ibadan, Nigeria; ${ }^{2}$ Department of Oral Pa- \\ thology, University of Ibadan and University College Hospital Ibadan, Ibadan, Nigeria; ${ }^{3}$ Department of Pathology, University of \\ Ibadan and University College Hospital Ibadan, Ibadan, Nigeria. \\ Email: "ginaoladokun@yahoo.com
}

Received June $23^{\text {rd }}$, 2011; revised July $11^{\text {th }}, 2011$; accepted July $20^{\text {th }}, 2011$.

\begin{abstract}
Background: HIV associated KS is relatively rare in children and has been reported to be higher in East Africa compared to other regions. Literature on cases of histologically proven Kaposi sarcoma in children with HIV infection in West Africa is scanty. Case presentation: This communication presents three cases of KS seen among children in a paediatric HIV unit. The first case was an eleven year old HIV positive boy who had oral candidiasis that resolved with treatment and subsequently developed a painless, erythematous swelling at the middle of the dorsum of the tongue with central loss of papillae. He also had multiple discoid hyperpigmented flat lesions on the legs and soles of the feet. In addition to switching to second line antiretroviral therapy, he had chemotherapy. The lesions regressed. The second case was a double orphan who had KS involvement of the right eye, nasal cavity and lymph nodes. The tumour rapidly progressed and the child died before ART and chemotherapy could be commenced. The last case was a five year old girl with mild KS of the skin but also had other manifestations of severe HIV disease which she succumbed to. Incisional biopsies of the lesions revealed an invasive epithelial lined vascular tumour destroying the upper layers of skeletal fibres within the connective tissue stroma in keeping with KS. Human Herpes Virus type 8 (HHV8) screen was also positive for all the cases. Conclusion: A high index of suspicion must be entertained and biopsy of suspicious muco-cutaneous lesions is necessary to exclude a diagnosis of KS which is an indicator of severity and progression of HIV.
\end{abstract}

Keywords: Kaposi Sarcoma, HIV Infection, Children, West Africa

\section{Introduction}

Though Kaposi Sarcoma (KS) is relatively infrequent among children compared to adults, in some parts of resource poor continents such as East Africa and Latin America, frequent lesions of KS have been documented among children and had been attributed to endemic presence of Human Herpes Virus type 8 (HHV8) [1,2]. HIV-associated Kaposi Sarcoma (HIV-KS) is the most common neoplasm observed in HIV-seropositive subjects [3] and it has been reported in different age groups of children worldwide presenting predominantly as mucocutaneous disease. The earliest documentation of childhood KS included a 6 day old neonate and a six month old infant both born to mothers with HIV/AIDS [4].

Although cutaneous lesions are the most common manifestations of KS [5], in 22\% of subjects with HIV KS, the lesion is first seen in the mouth and in $71 \%$ of the subjects with HIV KS the oral cavity will be affected. The most common intra oral site is the palate followed by the lips and the tongue [6]. Among children with HIV/AIDS in the developing world, the prevalence of oral lesions vary from country to country, but it is widely accepted that oral candidiasis is the most common lesion in children. By contrast, neoplastic lesions that involve the oral cavity including Kaposi sarcoma are relatively rare $[7,8]$.

What is known about the aetiology of KS has evolved. Viruses may play an important role in its aetiopathogenesis. The most convincing evidence for viral aetiology was proposed following the discovery of DNA sequences of HHV8 in KS lesions of virtually all AIDS patients [9]. HHV8 DNA is seen in higher concentration in oral fluids than other bodily secretions in subjects with HIV-KS and this may partly explain the high prevalence of HIV-KS in the mouth. In a retrospective study [10], it 
was observed that death rate in subjects with oral HIVKS was higher than in subjects with exclusive cutaneous manifestations [11]. Genetic predisposition was proposed by Friedman Kien et al. [12] as an aetiological factor which could be as a result of increased incidence of histocompatibility marker HLA-DR5 in $62 \%$ of classic KS while Windle-Taylor and Shah [13] suggested an environmental aetiology due to specific geographic distribution of the disease. Though HIV is not a direct aetiological agent, it provides the environment for opportunistic factors. Furthermore, other factors including HIV transactivating (tat) gene and cytokines such as Oncostatin M and scatter factor, along with some states of immunesystem compromise are important contributors to the development of KS [14].

There had not been any previous documentation of cases of clinical and histologically proven Kaposi sarcoma in children with HIV infection in Nigeria, $[15,16]$ This report was therefore necessary to document and to alert clinicians to the possibility of this diagnosis in HIV infected children.

\section{Case Presentation}

\subsection{Case 1}

This 11 year old HIV positive boy who had been on antiretroviral therapy (ART) for four months was admitted into a paediatric ward of the University College Hospital, Ibadan, Nigeria in June 2008. He was referred on the suspicion of HIV infection to the Hospital five and a half months earlier. The HIV rapid test was reactive and was confirmed by Western blot. HIV infection was also confirmed in his mother. He had presented with angular cheilitis and difficulty with eating, facial swelling, persistent cough, bilateral leg swelling, recurrent fever and generalised non-pruritic hyperpigmented skin lesions.

On examination, he was wasted with his weight at $68 \%$ of the median for age. His tongue showed a cream coloured plaque at the lateral aspect with erythematous margin that extended to the oropharynx. He was dyspnoeic and had lung crepitations. The chest radiograph showed homogenous opacities on the right mid zone but the Mantoux test and sputum microscopy for Acid Fast Bacilli were negative. At this stage, the haematocrit was 22\%; CD4 count 14 cells/ $\mu$ l and CD4\% 2.2. An abdominal ultrasound revealed normal sized kidneys with increased echogenicity and coarse echo texture without intrinsic masses.

Urinalysis showed a specific gravity of 1.012 and protein (2+), liver function tests revealed hypoproteinaemia and hypoalbuminaemia but the enzymes were within normal limits. He was diagnosed then with the World Health Organization (WHO) Clinical stage 4 HIV disease with right lobar pneumonia, oropharyngeal candidiasis (pseudomembranous type) and possible HIV Nephropathy. He commenced first line anti-retroviral therapy (zidovudine, lamivudine \& nevirapine) alongside antibiotics (cefuroxime and gentamycin) and antifungal agents (fluconazole). At the end of two weeks, the oropharyngeal lesion and lung crepitations had resolved and he was discharged and continued management on outpatient basis.

Two months later he presented with anorexia, recurrent vomiting and difficulty with walking. On examination, he was pale and had recent appearance of darkish brown to black multiple maculo-papular pigmented lesions on the trunk, lower extremities and a solitary nodular lesion on the plantar surface of the right foot (Figure 1(a)). There was also an intra-oral, erythematous swelling located centrally on the dorsum of the tongue measuring $3 \times 2 \mathrm{~cm}$ (Figure 1(b)). The patient admitted that swelling on the tongue was first noticed two weeks earlier and had increased gradually in size without associated pain. In view of the anatomical location, central loss of papillae and associated history of oropharyngeal candidiasis, an initial clinical impression of median rhomboid glossitis was entertained. This lesion however did not respond to antifungal therapy. An excisional biopsy of the tongue lesion was carried out. Special stain with Gomori's methenamine silver failed to demonstrate fungal hyphae. Haematoxylin and eosin stained histologic sections of both the tongue and skin lesions revealed a cellular neoplasm composed of spindle cells with slit-like spaces containing red blood cells (Figure 2(a)). There was deep skeletal muscle invasion by tumour cells, with chronic inflammation in the upper corium which was in keeping with KS (Figures 2(b) and 2(c)).

Additionally, the leg swelling had persisted and the serum creatinine became elevated. A renal biopsy was carried out and histology revealed moderate cystic dilation with mild degeneration of both proximal and distal tubules and focal segmental glomerulosclerosis. Based on these features the diagnosis of HIV associated nephropathy was reinforced. CD4 count and CD4\% at this point was 29 and $4.9 \%$ respectively while the haematocrit was $28 \%$.

The tumour cells showed strong granular nuclei staining in keeping with KS HHV-8 (Figure 2(d)). He was readmitted and switched to second line antiretroviral therapy (Lamivudine, Didanosine, Lopinavir/ritonavir). He was monitored for toxicity and subsequently chemotherapy was added to the treatment as stipulated in the National guideline for treatment of HIV associated KS [17] The chemotherapy regimen consisted of two weekly courses of Adriamycin for 6 cycles. Two months after the commencement of chemotherapy, the tongue 


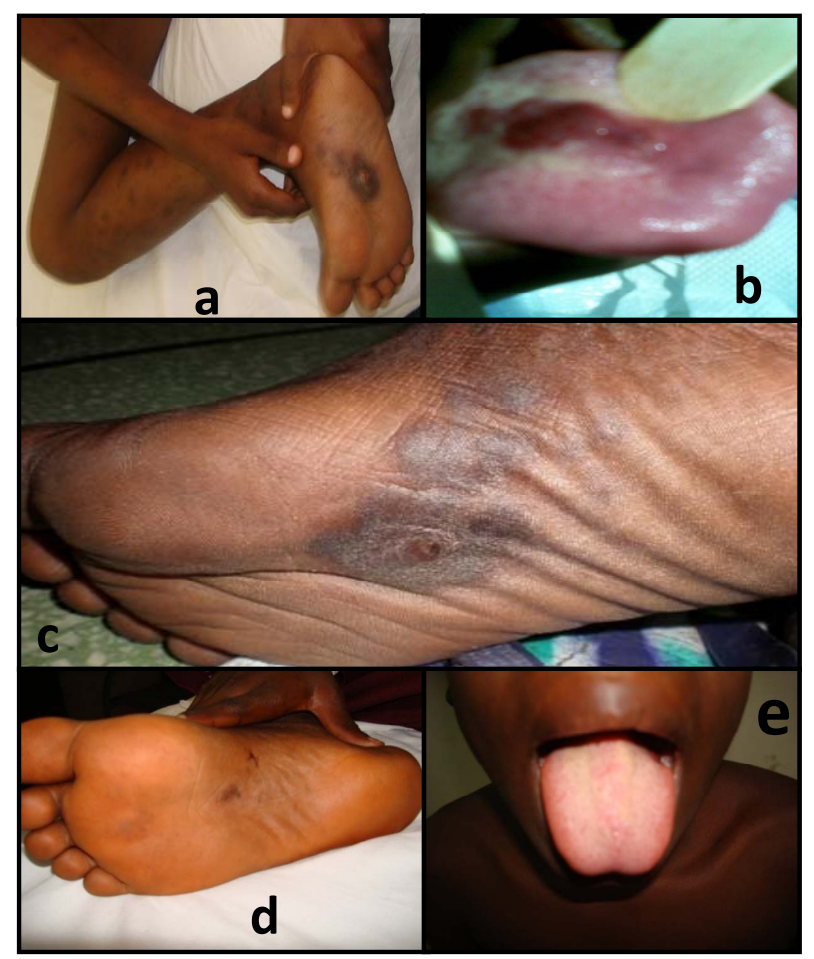

Figure 1. Case 1. (a) Initial nodular lesion on the plantar surface of the right foot; (b) Initial multiple coalescent nodular haemorrhagic tongue lesions; (c) Plantar lesion 2 months after commencing chemotherapy; (d) KS lesion on sole of right foot, post chemotherapy; (e) KS lesion on the tongue post treatment.

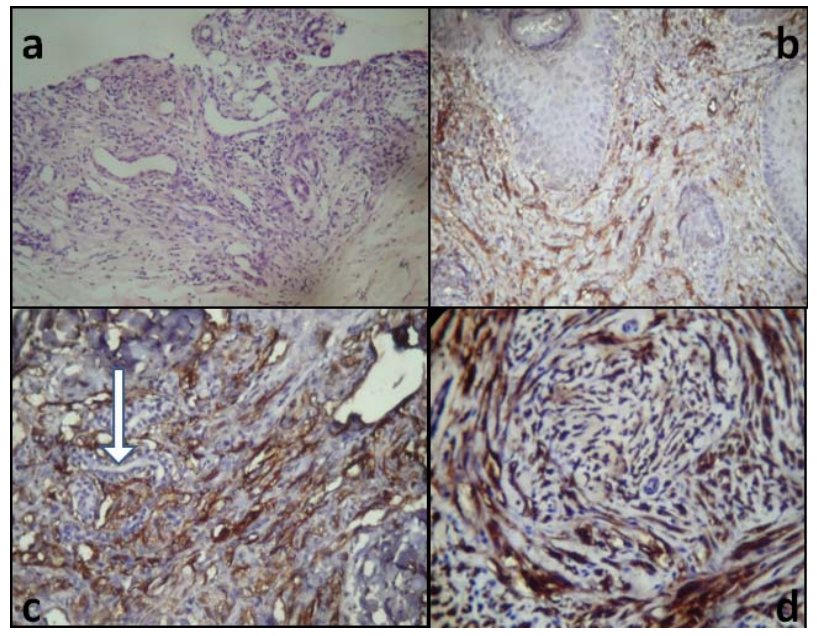

Figure 2. Histology and KS HHV-8 staining of tumour cells. (a) Haematoxylin and eosin section $(\times 200)$ show superficial dermal vascular and spindle cell proliferation; (b) Prominent staining of the vascular endothelia lining with CD 31 and involvement of the superficial dermis (× 200); (c) CD 34 positive endothelial vascular channel of a dermal KS ramifying around a sweat gland-block arrow (× 200); (d) KS HHV-8 staining of the tumour cells with strong granular nuclei staining $(\times 400)$. lesion had regressed completely (Figure 1(c)) and the plantar lesion had also flattened (Figure 1(d)) and was no longer tender. At the time of this report, nearly three years after, the tongue and skin lesions had not recurred (Figures 1(d) and 1(e)) and the serum electrolyte, urea and creatinine are normal. He is regular on second line antiretroviral therapy and has since achieved complete viral suppression $(<200$ copies/ml) with the latest CD4+ count of 760 cells/ $\mu$ l.

\subsection{Case 2}

This was a 9 year old boy that presented in February, 2009 at the same health facility with cough of 3 months duration, neck and scalp swellings and protrusion from the right eye of 2 months duration. His parents had died of wasting disease within the previous 6 months before his presentation. The causes of death were not confirmed before demise. The patient had two siblings; the youngest had died in infancy while the immediate younger sibling was alive. Both surviving children were living with their maternal grandmother.

The swellings over the scalp and neck progressively increased in size. The growth on the right eye was painless with associated watery eye discharge and gradual loss of vision.

At presentation, he was wasted, small for age and had extensive scaly lesions on the scalp. He was mildly pale, afebrile, anicteric, had generalized lymphadenopathy and no peripheral oedema. He had a fungating nodular mass protruding from the right eye and other nodular swellings on the right temporal region (Figures 3(a)-(c)) and lower limbs. His weight was $17 \mathrm{~kg}$ (58.6\% of expected) and his height was $111 \mathrm{~cm}$ being 84.7 per cent of the median for his age indicative of stunting.

Significant findings on physical examinations included noisy breathing, intercostal recessions with a respiratory rate of 36 breaths per minute, reduced air entry on the left lower lung zone, bronchial breath sounds on the right upper and mid lung zones. There was hepatomegaly of 4 $\mathrm{cm}$ below the right costal margin.

The chest radiograph showed collapse of the right upper lobe with compensatory emphysema of the right middle and lower lobes and the left lung. Mantoux test and sputum microscopy for Acid Fast Bacilli were negative. His haematocrit was $19 \%$ and serum electrolytes, urea and creatinine were within normal limits. Rapid HIV test was reactive and was confirmed by Western Blot. His CD4 count was 72 cells/ul (0.2\%) and viral load was 168,041 copies/ml. He tested positive to Hepatitis $C$ antibody and negative to Hepatitis B surface antigen.

He was diagnosed to have WHO clinical stage 4 HIV Infection with possible disseminated Tuberculosis (pulmonary, abdominal, lymph node), Kaposi Sarcoma and 


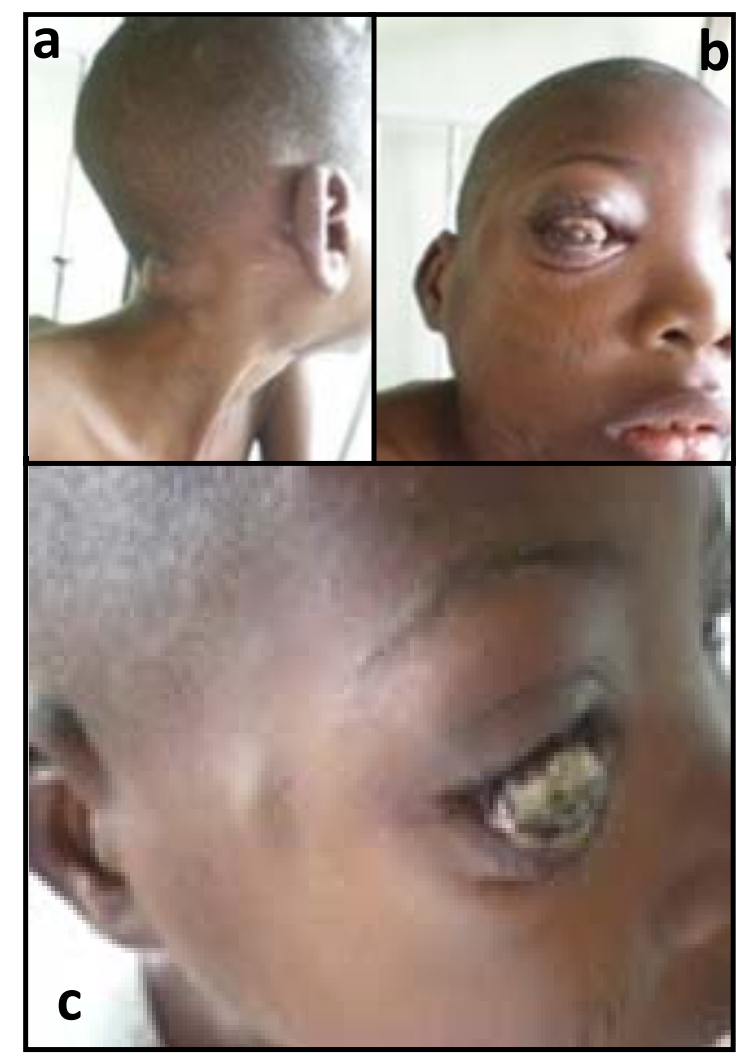

Figure 3. CASE 2. (a) Nodular anterior and posterior cervical neck swellings; (b) Exopthalmos of the right eye; (c) Corneal tumour and nodular swelling on the right temporal region.

tinea capitis. He received antibiotics for suspected pyogenic pneumonia, ketoconazole, Cotrimoxazole for treatment of Pneumocystis pneumonia and anti tuberculosis therapy. ART was commenced two weeks later. However, his clinical status continued to deteriorate with worsening respiratory distress and nodular lesions extending to the left eye, left nostril, palate and the upper limbs that bled to touch. Histology of the lymph node and skin nodule were in keeping with KS and the HHV8 screen was also positive.

While he was being worked up for chemotherapy; he developed copious serosanguinious discharges from the nostrils and petechiae haemorrhages on the skin. He went into cardiopulmonary arrest for which resuscitative efforts were unsuccessful.

\subsection{Case 3}

This was a five year old girl who presented to the UCH, Ibadan in September 2010 with a 3 month's history of recurrent fever, cough, weight loss and diarrhoea. Her weight was $11 \mathrm{Kg}$ (60\% of expected) and the height was $105 \mathrm{~cm}$ ( $50^{\text {th }}$ percentile). The mid upper arm circumference was $11.2 \mathrm{~cm}$. She was chronically ill with florid oral thrush, angular stomatitis, generalized lymphadenopathy, crepitations in the lungs and hepatomegaly. HIV infection was confirmed by Western Blot. Her mother and younger sibling were also confirmed to have HIV infection. Her CD4 count was 4 cells/ $\mu$ l (1.4\%). She was transfused with packed red cells on account of a haematocrit of $11 \%$. She also had severe hypokalaemia (1.9 mmol/l) which was corrected and symptomatic hypocalcaemia that manifested as carpopedal spasm that resolved with calcium therapy. Haemoglobin genotype could not be ascertained as she had received blood transfusion shortly before her presentation to UCH. An initial chest radiograph was normal, mantoux test was negative, and gastric washings for AFB was also negative. ART was commenced two weeks later using a combination of Zidovudine, Lamivudine and Efavirenz. She also received nutritional rehabilitation with ready to use foods. As a result of her continued poor clinical status and failure to improve on antibiotics, a repeat chest radiograph later showed bilateral opacities on account of which she was treated with antituberculosis therapy consisting of Rifampicin, Isoniazid, Pyrazinamide and Ethambutol.

Four weeks into admission, two hyperpigmented nodular non-tender lesions measuring $1.5 \mathrm{~cm}$ in the widest diameter were observed over the left temporal region of the face which was histologically confirmed as Kaposi sarcoma (Figure 4). The KS HHV8 screen was also positive. She had minimal improvement in her general condition initially but later deteriorated with development of jaundice and an episode of generalized tonicclonic seizure. The cerebrospinal fluid analysis was not suggestive of meningitis or meningoencephalitis. On account of suspected drug toxicity the antituberculosis and antiretroviral drugs were discontinued. However she died shortly after while further investigations into this were on going.

\section{Discussion}

These cases of histological proven Kaposi sarcoma in these children with confirmed HIV infection in this report illustrate that KS occurs in children not only in East Africa [6] and Southern America [4], but in other parts of Africa including Nigeria. In a study of AIDS associated KS in Nigeria over a 10 year period, it was reported that there was no case of childhood involvement among the patients [18]. This was thought to be from misdiagnosis as KS may be confused with lesions including congenital haemangioma, dysplastic naevi and dermatofibromas. Routine tissue biopsy of suspicious lesions in children would increase the detection of KS among HIV positive children with skin lesions. The three cases in this report had KS involvement of the skin. However, KS may also affect the less obvious organs in the body and also affect 


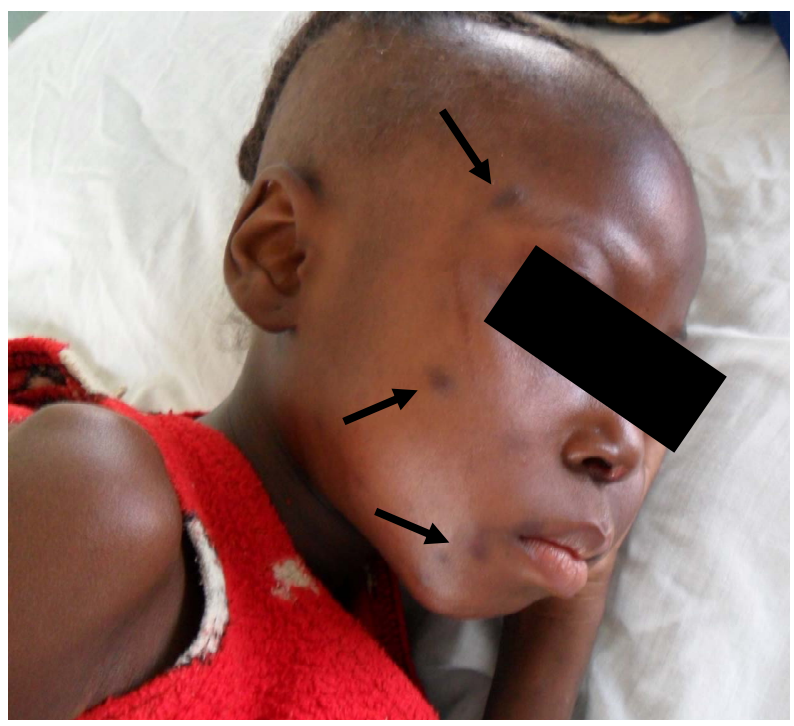

Figure 4. CASE 3. Hyper pigmented nodular lesions on the right temporal, maxillary and mandibular regions indicated with black arrows.

multiple sites. Multiple sites of KS were seen in almost $45 \%$ of the children with HIV and AIDS with the skin being the most common site, occurring either alone or in combination with other sites. The skin was involved in over $57 \%$ of the children in the series reported from South Africa [19]. In addition, as a result of the high burden of tuberculosis and other opportunistic infections, KS of the lungs may be misdiagnosed [20]. The issue is further complicated by the fact the chest radiograph findings may be unspecific as it could mimic a myriad of other infectious and non infectious diseases. Radiographic findings of pulmonary Kaposi's sarcoma have been described in HIV positive children. The typical features were reported to include hilar lymphadenopathy, diffuse bilateral peri-hilar and lower zone infiltration, air space and reticular opacification, and effusions [21]. The second case in our report may well have had pulmonary involvement of KS. This could not be confirmed as a post mortem could not be carried out.

The children in this report were all older than 5 years of age and the mode of transmission of HIV was presumably vertical as they had no other obvious risk factors for HIV infection. Though the onset of HIV symptoms was delayed, the progression of the disease was rapid. Other reports of KS in the paediatric age group were also in older children who had had long standing disease [22]. Children known to have had prior blood transfusions probably resulting in HIV infection had also been reported to have KS and did not have features that might distinguish them from those who acquired HIV from their mothers [2]. It is to be noted also that our patients had severe immunosuppression with very low CD4+ counts. In a larger retrospective paediatric study, a similar observation was made where all 61 but two cases had a severely compromised immunity [19].

Candidiasis is the most frequent oral feature of HIV/ AIDS in children and some authors have separated cases presenting with angular cheilitis or pseudomembranous features from other variants [22,23]. The first case presented had pseudomembranous type in the oropharynx which responded to fluconazole an antifungal agent. Due to the previous episode of oropharyngeal candidiasis and the anatomical location of the tongue lesion, an initial impression of median rhomboid glossitis was entertained but special stain with Gomori's methenamine silver failed to demonstrate fungal hyphae. Other differential diagnoses of depapillated midline nodular tongue swellings include lingual thyroid, gumma of tertiary syphilis, granuloma of tuberculosis and granular cell tumour. All the above were excluded with the outcome of the tissue biopsy of the tongue that had been described earlier. The histological features coupled with the skin lesions that became more obvious led to diagnosis of Kaposi's sarcoma.

Although neoplasms are rare oral manifestations of children infected with HIV/AIDS [24], the tendency to develop oral KS and oral hairy leukoplakia has been known to increase with age due to longer exposure to HHV-8 and EBV [25]. The three children in the study had evidence of HHV-8 co-infection. The pathomechanism of development of KS following HSV infection is uncertain but it is postulated that HHV8 encodes several genes that have capacity to stimulate angiogenesis by inducing angiogenic phenotype transformation of HHV-8 infected cells or by production of inflammatory cytokines, chemokines and growth factors that can activate and promote angiogenesis [5]. In a study of KS in childhood in Uganda, immune dysfunction and local inflammation were implicated as predisposing factors since prodromal symptoms of HIV infection were present in the majority and $39 \%$ of patients had an infection in a site later involved with KS [2].

Patients with KS need to be diagnosed and treated with ART promptly in order to reduce mortality as survival correlates significantly with the antiretroviral treatment [19]. A challenge in the management of these patients would be monitoring for toxicities that could result from drug interactions from the combination of antiretroviral therapy, anti tuberculosis regimen, chemotherapy, antifungals and other drugs that may be required. A twofold risk of developing opportunistic infections had been reported in HIV infected patients with Non-Hodgkin's lymphoma who had been treated with systemic chemotherapy [26]. The risk for opportunistic infections in HIV-infected patients with KS is also increased while 
receiving chemotherapy [27]. In many studies which were mainly in adults, Kaposi's sarcoma Immune Reconstitution Inflammatory Syndrome (IRIS) had also been reported $[28,29]$.

\section{Conclusions}

KS occurs in children with HIV infection in Nigeria, Western Africa though it appears to be uncommon when compared with the occurrence in some other African sub-regions. With the increased availability of antiretroviral therapy, which is associated with better survival, there should be a high index of suspicion for KS in HIV infected children with suggestive signs as its presence is an indication of severity and progression of the disease. Biopsy of such lesions in muco-cutaneous and other accessible sites is necessary to exclude a diagnosis of KS. Early diagnosis for prompt institution of therapy is necessary to improve the chances of survival.

\section{REFERENCES}

[1] K. Ranganathan and R. Hemalatha, "Oral Lesions in HIV Infections in Developing Countries: An overview,” Advances in Dental Research, Vol. 19, No. 1, 2006, pp. 6368. doi:10.1177/154407370601900113

[2] J. L. Ziegler and E. Katongole-Mbidde, “Kaposi’s Sarcoma in Childhood: An Analysis of 100 Cases from Uganda and Relationship to HIV Infection,” International Journal of Cancer, Vol. 65, No. 2, 1996, pp. 200-203. doi:10.1002/(SICI)1097-0215(19960117)65:2<200::AIDIJC12>3.0.CO;2-H

[3] L. Feller and J. Lemmer, "HIV-Associated Kaposi’s Sarcoma: Pathogenic Mechanisms,” Oral Surgery Oral Medicine Oral Pathology Oral Radiology and Endodontics, Vol. 104, No. 4, 2007, pp. 521-529. doi:10.1016/j.tripleo.2006.08.015

[4] K. P. Manji, H. Amir and I. Z. Maduhu, "Aggressive Kaposi's Sarcoma in a 6-Month-Old African Infant: Case Report and Review of the Literature,” Tropical Medicine \& International Health, Vol. 5, No 2, 2000, pp. 85-87. doi:10.1046/j.1365-3156.2000.00523.x

[5] A. K. Agarwal, G. Zamzachin, Y.Mohen, M. Y. Singh and N. Singh, "AIDS Related Kaposi's Sarcoma-Like Lesion,” Indian Journal of Dermatology, Vol. 62, No. 2, 1996, pp. 129-131.

[6] J. B. Epstein, R. J. Cabey and M. Glick, “Oral Malignancies in HIV Disease: Changes in Disease Presentation, Increasing Understanding of Molecular Pathogenesis and Current Management," Oral Surgery Oral Medicine Oral Pathology Oral Radiology, and Endodontics, Vol. 100, No. 5, 2005, pp. 571-578. doi:10.1016/j.tripleo.2005.01.015

[7] P. Khongkunthlan, M. Grote, W. Isaratanan, S. Plyaworawong and P. A. Reichart, "Oral Manifestations in 45 HIV-Positive Children from Northern Thailand," Journal of Oral Pathology \& Medicine, Vol. 30, No. 9, 2001, pp.
549-552. doi:10.1034/j.1600-0714.2001.300907.X

[8] I. D. Miziara, B. C. Araújo Filho and R. Weber, "Oral Lesions in Brazilian HIV-Infected Children Undergoing HAART," International Journal of Pediatric Otorhinolaryngology, Vol. 70, No. 6, 2006, pp. 1089-1096. doi:10.1016/j.ijporl.2005.11.010

[9] I. Lager, M. Altini, H. Coleman and H. Ali, “Oral Kaposi's Sarcoma: A Clinicopathologic Study from South Africa," Oral Surgery Oral Medicine Oral Pathology Oral Radiology, and Endodontics, Vol. 96, No. 6, 2003, pp. 701-710. doi:10.1016/S1079-2104(03)00370-6

[10] M. M. Taylor, B. B. Chohan, L. Lavreys, W. Hassan, M. L. Huang, L. Corey, R. Ashley Morrow, B. A. Richardson, K. Mandaliya, J. Ndinya-Achola, J. Bwayo and J. Kreiss, "Shedding of Human Herpes Virus 8 in Oral and Genital Secretions from HIV-1 Seropositive and Seronegative Kenyan Women,” Journal of Infectious Diseases, Vol. 190, No. 3, 2004, pp. 484-488. doi:10.1086/421466

[11] B. Rohrmus, E. M. Thoma-Greber, M. Rocken and J. R. Bogner, "Outlook in Oral and Cutaneous Kaposi's Sarcoma,” Lancet, Vol. 356, No. 9248, 2000, p. 2160. doi:10.1016/S0140-6736(00)03503-0

[12] A. E. Friedman-Kien, L. J. Laubenstein, P. Rubinstein, E. Buimovici-Klein, M. Marmor, R. Stahl, I. Spigland, K. S. Kim and S. Zolla-Pazner, "Disseminated Kaposi's Sarcoma in Homosexual Men,” Annals of internal medicine, Vol. 96, No. 6, 1982, pp. 693-700.

[13] P. C. Windle-Taylor and N. Shah, "Oropharyngeal Kaposi's Sarcoma: Report of Two Cases and Review of the Literature,” Journal of Laryngology and Otology, Vol. 97, No. 11, 1993, pp. 1065-1071.

[14] C. Oji and F. Chukwuneke, "Clinical Evaluation of Kaposi Sarcoma in HIV/AIDS Patients with Orofacial Lesions in Enugu, Nigeria,” Journal of Oral Maxillofacial Surgery, Vol. 66, No. 7, 2008, pp. 1362-1365. doi:10.1016/j.joms.2007.08.049

[15] M. I. Adurogbangba, G. A. Aderinokun, G. N. Odaibo, O. D. Olaleye and T. O. Lawoyin, "Oro-Facial Lesions and CD4 Counts Associated with HIV/AIDS in an Adult Population in Oyo State, Nigeria,” Oral Diseases, Vol. 10, No. 6, 2004, pp. 319-326. doi:10.1111/j.1601-0825.2004.01036.x

[16] G. A. Agbelusi and A. A. Wright, "Oral Lesions as Indicators of HIV Infection among Routine Dental Patients in Lagos, Nigeria,” Oral Diseases, Vol. 11, No. 8, 2005, pp. 370-373.

[17] Federal Ministry of Health of Nigeria, "National Guidelines on Cancer Chemotherapy," Federal Ministry of Health of Nigeria, 2008.

[18] N. Mbah, I. H. Abdulkareem and A. Panti, “AIDS-Associated Kaposi’s Sarcoma in Sokoto, Nigeria,” Nigerian Journal of Clinical Practice, Vol. 11, No. 3, 2008, pp. 181-184.

[19] D. C. Stefan, D. K. Stones, L. Wainwright and R. Newton, "Kaposi Sarcoma in South African Children," Pediatric Blood Cancer, Vol. 56, No. 3, 2011, pp. 392-396. doi:10.1002/pbc. 22903 
[20] A. Mosam, J. Aboobaker and F. Shaik, "Kaposi's Sarcoma in Sub-Saharan Africa: A Current Perspective," Current Opinion in Infectious Diseases, Vol. 23, 2010, pp. 119-123.

[21] S. Theron, S. Andronikou, R. George, J. du Plessis, P. Goussard, M. Hayes, A. Mapukata and R. Gie, "Non-Infective Pulmonary Disease in HIV-Positive Children," Pediatric Radiology, Vol. 39, No. 6, 2009, pp. 555-564. doi:10.1007/s00247-009-1156-2

[22] C. Okunseri, V. Badner, A. Wiznia and M. Rosemberg, "Prevalence of Oral lesions and Percent CD41+ T-LymPhocytes in HIV Infected Children on Antiretroviral Therapy,” AIDS Patient Care and STDS, Vol. 17, No. 1, 2003, pp. 5-11. doi:10.1089/108729103321042863

[23] A. Chan, A. Milnes, S. M. King and S. Read, "The Relationship of Oral Manifestations to Parameters of Immune Function and CDC Stage in Children Born to HIV Positive Women," In: Pediatric AIDS and HIV Infection: Fetus to Adolescent, Williams \& Wilkins, Baltimore, 1994, pp. 101-107.

[24] B. Ensoli, R. Gendelman, P. Markham, V. Fiorelli, S. Colombini, M. Raffeld, A. Cafero, H. K. Chang, J. N. Brady and R. C. Gallo, "Synergy between Basic Fibroblast Growth Factor and HIV-1 Tat Protein in Induction of Kaposi's Sarcoma,” Nature, Vol. 371, 1994, pp. 674680. doi:10.1038/371674a0

[25] M. W. Kline, “Oral Manifestations of Pediatric Human
Immunodeficiency Virus Infection: A Review of the Literature,” Pediatrics, Vol. 97, No. 3, 1996, pp. 380-388.

[26] J. A. Sparano, X. Hu, P. H. Wiernik, C. Sarta, D. M. Reddy, L. Hanau and D. H. Henry, "Opportunistic Infection and Immunologic Function in Patients with Human Immunodeficiency Virus-Associated Non-Hodgkin's Lymphoma Treated with Chemotherapy," Journal of the National Cancer Institute, Vol. 89, No. 4, 1997, pp. 301-307. doi:10.1093/jnci/89.4.301

[27] A. Plettenberg, U. van Dyk, A. Stoehr, H. Albrecht, H. J. Stellbrink, J. Berger and W. Meigel, "Increased Risk for Opportunistic Infections during Chemotherapy in HIV-Infected Patients with Kaposi's Sarcoma,” Dermatology, Vol. 194, No. 3, 1997, pp. 234-237. doi:10.1159/000246109

[28] D. M. Murdoch, W. D. Venter, C. Feldman and A. Van Rie, "Incidence and Risk Factors for the Immune Reconstitution Inflammatory Syndrome in HIV Patients in South Africa: A Prospective Study,” AIDS, Vol. 22, No. 5, 2008, pp. 601-610. doi:10.1097/QAD.0b013e3282f4a607

[29] E. Letang, J. Almeida, E. Ayala, I. E. White, C. Carrilho, R. Bastos, T. Nhampossa, C. Menéndez, T. B. Campbell, P. L. Alonso and D. Naniche, "Predictors of Immune Reconstitution Inflammatory Syndrome-Associated with Kaposi Sarcoma in Mozambique: A Prospective Study," Journal of Acquired Immune Deficiency Syndrome, Vol. 53, No. 5, 2010, pp. 589-597. 